\title{
Development of sustainable concrete repair materials via microencapsulated agents
}

\author{
Chrysoula Litina ${ }^{1 *}$ and Abir Al-Tabbaa ${ }^{1}$ \\ ${ }^{1}$ Engineering Department, University of Cambridge, Trumpington Street, Cambridge CB2 1PZ, UK
}

\begin{abstract}
Identification of non-structural damage in concrete infrastructure and actuation of preventive repair solutions is an established approach to avoid further structural damages and more expensive repair regimes. However the repair of concrete itself is not infallible with 55\% of reported repairs in the EU failing within 5 years of service. Thus the already once repaired concrete structure is then subject to a constant cycle of repeated repair and a cumulative associated life cycle cost. The development of external repair material with self-healing capabilities, can affect a real step-change on the life-cycle costs and maintenance of existing and new infrastructure. Developed polymeric microcapsules containing liquid sodium silicate were used to impart autonomic self-healing to readily available commercial repair mortars for the first time. These materials cover a range of potential real time repair applications. Initially the compatibility between the developed self-healing agents and commercial products was established and the self-healing performance of the novel composite system was then evaluated. The results underlined the huge potential for the proposed composite systems as a stepping stone toward commercial uptake of self-healing microcapsule-based cementititious materials.
\end{abstract}

\section{Introduction}

Identification and correct diagnosis of the underlying cause of damage in concrete is the first step towards a reliable and durable repair strategy [1]. Although adequate design and selection of appropriate materials is a prerequisite, it is not the only limiting factor for robust repairs. To tackle this, most previous efforts have focused on improving the repair material performance and reducing or controlling the expected damage. These attempts can only result in marginal improvements as they do not address the underlying problem, the inherently brittle behaviour of concrete and its natural propensity to crack. Self-healing design can be a promising solution to prolong the functionality of a repaired section by imbuing repair materials with a built-in capability to repair inflicted damage.

Self-healing in concrete is an acknowledged intrinsic behaviour occurring autogenously through a combination of physical, chemical and mechanical processes [2]. Yet the phenomenon can be erratic and its efficiency limited since a number of factors need to be met for its occurrence. The phenomenon has been investigated extensively and different healing systems to enhance and benefit from this behavior have been put forward over the last twenty years; including expansive mineral additives, super absorbent polymers, microcapsules, bacterial carbonate precipitation and microfibers [3-7]. Amongst these technologies the encapsulation approach, namely the embedment of repair agents in vessels within the matrix to be released at the location and time of damage and actuate repair on demand is most promising.

A particular category of encapsulation is microencapsulation. The principle of self-healing through microencapsulation is that microcapsules are embedded in the bulk of the host matrix during manufacture and trigger the release of their healing compound upon rupture of their shell $[8,9]$. The latter is initiated by stress built-up during crack formation. Chemical interactions between the released core material(s) and the surrounding matrix heal the crack restoring partially or fully the bulk material properties. Such a system that does not require external trigger, poses no restriction to normal casting and can address multiple simultaneous cracking offers the greatest flexibility for field applications and direct incorporation in ubiquitous repair materials.

More recently collaborative research at the University of Cambridge [10] led to the development of polymeric microcapsules that contain a liquid sodium silicate core. The healing potential of the microcapsules under different cracking regimes was confirmed [11] and the effect of these healing additives was investigated on both fresh (viscosity - setting time) and hardened properties (modulus of elasticity, compressive and flexural strengths) $[12,13]$. The potential of the developed microcapsule-based concrete material was demonstrated in new concrete elements with full-scale field trials $[14,15]$ but also assessed for use in existing infrastructure as a repair material.

This paper focuses on the latter reporting on the development of microcapsule based self-healing concrete

\footnotetext{
* Corresponding author: c1519@cam.ac.uk
} 
materials for reliable concrete repairs. Different commercially available products covering a range of potential real time repair applications were targeted; including materials for cementitious hand-placed, poured and sprayed repairs, structural and non-structural. An initial objective was to establish compatibility between the developed healing-promoting microcapsules and the commercial products. Subsequently the potential of the novel composite system for self-healing applications was assessed and established.

\section{Experimental Methodology \\ 1. Materials and mixture proportions}

Five repair mortars were selected for this project provided by Fosroc Ltd; a high build repair mortar (HBRM), a dry spray repair mortar (DSRM), a low alkali repair mortar (LARM), a patching repair mortar (PRM) and a selflevelling underlayer repair mortar (SLRM). All materials were provided as single component, packed products that only required the addition of a predetermined amount of water. For comparison, an overview of the compositions, in terms of their Portland cement blends, is presented in Fig. 1.

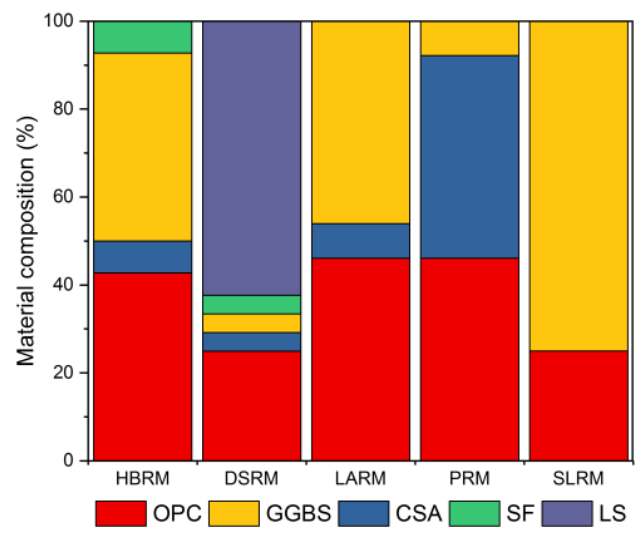

Fig. 1. Relative range of cementitious material composition of commercial repair mortars considered in this investigation as provided by the manufacturer. LS: limestone, SF: silica fume, GGBS: ground granulated blast furnace slag, CSA: calcium sulphoaluminate cement, OPC: ordinary Portland cement.

The self-healing additives used in this study were microcapsules developed previously by Lambson in collaboration with the University of Cambridge [10]. The microcapsules have a polymeric shell made of crosslinked gelatine and gum arabic and their cargo material consists of a solution of mineral oil and liquid sodium silicate $\left(40 \%\right.$ weight in $\left.\mathrm{H}_{2} \mathrm{O}\right)$ and they have an average size of $290 \mu \mathrm{m}$ and a density of $\sim 1.0 \mathrm{~g} / \mathrm{cm}^{3}$. Images of the microcapsules are shown in Fig. 2. Sodium silicate was selected, as the healing compound, because it can form products of similar characteristics to the host cementitious matrix. The potential for aqueous sodium silicate as a healing agent in cement-based materials has previously been illustrated both in terms of crack closure and durability [13,16-20].

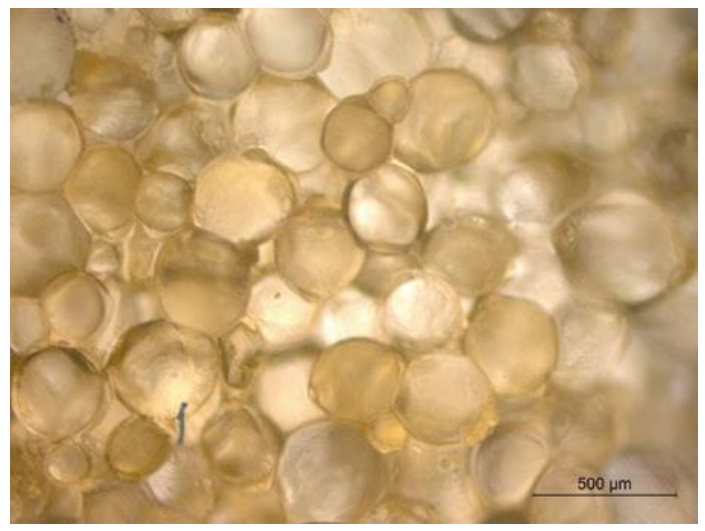

Fig. 2. Microscope images of microcapsules used in this study.

Considering previous results of microcapsule content on fresh and hardened material properties and in terms of healing potential $[11,13]$, three different microcapsule contents were proposed within the so far investigated range as part of the compatibility testing; $8 \%, 16 \%$ and $32 \%$ by volume of cement.

\section{Experimental programme}

Fresh properties such as setting time and workability were tested to ensure compatibility and suitability for practical applications, while mechanical properties were tested in terms of compressive and flexural strengths. These were determined to be the more critical elements for compliance with BS EN 1504. Tests were conducted at 1, 7 and 28 days for all mixes.

Optimum self-healing additive fractions were determined and further investigation on the in situ selfrepair potential of these novel materials was carried out. These tests included the evaluation of both mechanical integrity and durability recovery potential through threepoint bending and sorptivity testing. Seven and 28 days of initial cracking were selected to establish the healing efficiency of the composite material. Cracked samples were placed in high relative humidity $(\sim 68 \%)$ containers to allow healing to take place for 28 days. The latter condition was selected as both the most representative for real-time field applications and for conservative selfhealing estimation. To monitor crack initiation, opening and closure of the crack faces in all the specimens were monitored over time using a stereo microscope. A Leica S6D digital stereoscope was used. Digital images were captured at three different positions on the crack face. The cracks were photographed on the day of the cracking and at the end of the healing period at the exact same locations. Image analysis software (Image J) was then used to analyse the acquired captions and the average crack opening was calculated for each case. The values obtained from the image analysis were used to quantify the crack mouth healing percentage $(\mathrm{CMH})$ in each case as shown in Equation 2: 


$$
\operatorname{CMH}(\%)=\frac{w_{i}-w_{h}}{w_{i}} \times 100 \%
$$

Where $w_{i}$ is the initial crack width and $w_{h}$ is the healed crack width. To complement the macroscopic observations of the crack healing an Proceq ultrasonic pulse velocity test instrument was used for the determination of the evolution of the crack depth with healing time for each prismatic sample following the guidelines in BS-1881: Part 203. Probes were both placed on either side of the crack at a certain distance $x$ from the crack for the first measurement and $2 \mathrm{x}$ from the crack for the second measurement. The time required for the ultrasonic wave to travel between these two points was recorded and the crack depth $d$ was calculated as follows:

$$
d=x \sqrt{\frac{4 t_{1}^{2}-t_{2}^{2}}{t_{2}^{2}-t_{1}^{2}}}
$$

Where $\mathrm{x}$ is the distance between the probe location and the crack ( $30 \mathrm{~mm}$ in this case) and $t_{1}$ and $t_{2}$ is the time taken for the ultrasonic wave to travel through the concrete for the first and second measurement respectively. Tables 1 and 2 provides an overview of the investigated properties during both preliminary compatibility and self-healing performance stages.

Table 1: The investigated compatibility properties and testing protocols using microcapsule dosages of $0,8,16$ and $32 \%$ by volume.

\begin{tabular}{llll}
\hline Properties & & $\begin{array}{l}\text { Testing } \\
\text { protocols }\end{array}$ & Standard \\
\hline & $\begin{array}{l}\text { Compressive } \\
\text { strength }\end{array}$ & $\begin{array}{l}40 \times 40 \times 40 \\
\text { mm at 1, 7 } \\
\text { and 28 days }\end{array}$ & EN 196-1 \\
& & $\begin{array}{l}40 \times 40 \times \\
160 \mathrm{~mm} \text { at }\end{array}$ & EN 196-1 \\
Mechanical & Flexural & 1, 7 and 28 \\
properties & strength & & \\
& & & EN 1015-3 \\
& &
\end{tabular}

\footnotetext{
*In all experimental procedures triplicate

samples were used
}

Table 2: The investigated self-healing properties and testing protocols.

\begin{tabular}{lll}
\hline Properties & & Testing protocols \\
\hline $\begin{array}{l}\text { Mechanical } \\
\text { properties }\end{array}$ & $\begin{array}{l}\text { Flexural strength } \\
\text { width at maximum } \\
\text { recovery }\end{array}$ & $\begin{array}{l}\text { flexural loading } \\
\text { capacity, @7 and 28 } \\
\text { days (28 days } \\
\text { healing) }\end{array}$ \\
\hline & & $\begin{array}{l}\text { Controlled crack } \\
\text { width at maximum } \\
\text { flexural loading } \\
\text { capacity, @7 and 28 } \\
\text { days (28 days } \\
\text { healing) }\end{array}$
\end{tabular}

\begin{tabular}{lll}
\hline & $\begin{array}{l}\text { Ultrasonic wave } \\
\text { transmission } \\
\text { (crack depth) }\end{array}$ & $\begin{array}{l}\text { @7,14 and 28 days } \\
\text { (28 days healing) }\end{array}$ \\
\hline $\begin{array}{l}\text { Macroscopic } \\
\text { observations }\end{array}$ & $\begin{array}{l}\text { Crack area } \\
\text { measurements }\end{array}$ & $\begin{array}{l}\text { Optical microscopy } \\
\text { @7,14 and 28 days } \\
\text { (28 days healing) }\end{array}$ \\
\hline $\begin{array}{l}\text { Microscopic } \\
\text { observations }\end{array}$ & $\begin{array}{l}\text { Scanning electron } \\
\text { microscopy }\end{array}$ & \\
\hline *In all experimental procedures triplicate samples were used
\end{tabular}

\section{Results and Discussion}

\subsection{Compatibility with commercial repair mortars}

The inclusion of microcapsules into the mix did have an effect on the viscosity of the mix, in some cases substantial (Fig. 3). PRM and LARM appeared more sensitive to the addition of the healing agent to their composition. That effect became more pronounced as the microcapsule dosage was increased. Then the maximum increase in viscosity when compared to the control mix was more than $20 \%$. This affected the level and quality of compaction and hence the performance of the hardened material as confirmed by compressive strength results. However, the compressive strength did not show a consistent decrease with increasing concentration of microcapsules (Fig. 4). Although there was an observed loss of strength at 1 day, the 28 days' values for compressive strength for samples with microcapsules were for some repair materials higher than the control mixes prepared without self-healing additives. HBRM, DSRM and SLRM seemed to benefit from the presence of the microcapsules with higher strength achieved compared to the control- at 28 days for $8 \%$ microcapsule addition. In fact DSRM exhibited comparable strength to the reference specimen even with $32 \%$ by volume fraction addition. For these three materials the late development of strength compensates for the lower early age strength.

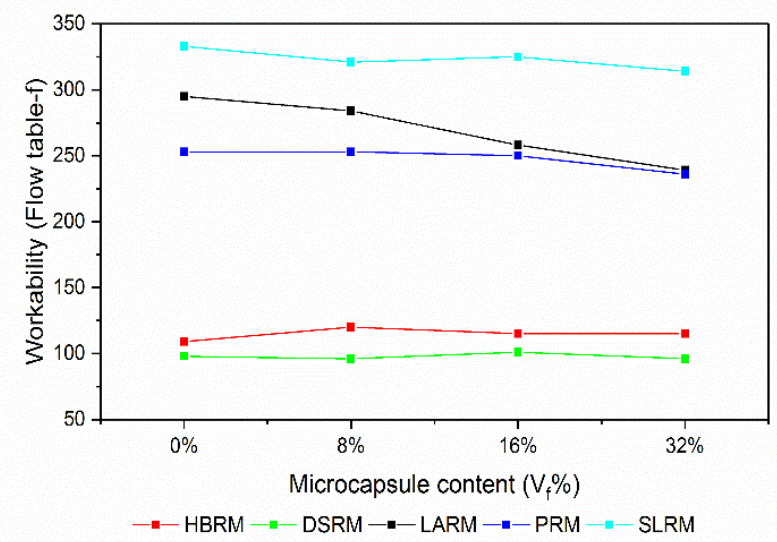

Fig. 3: Workability (flowtable results) variation with increasing loading percentage of microcapsules with respect to cement volume. 

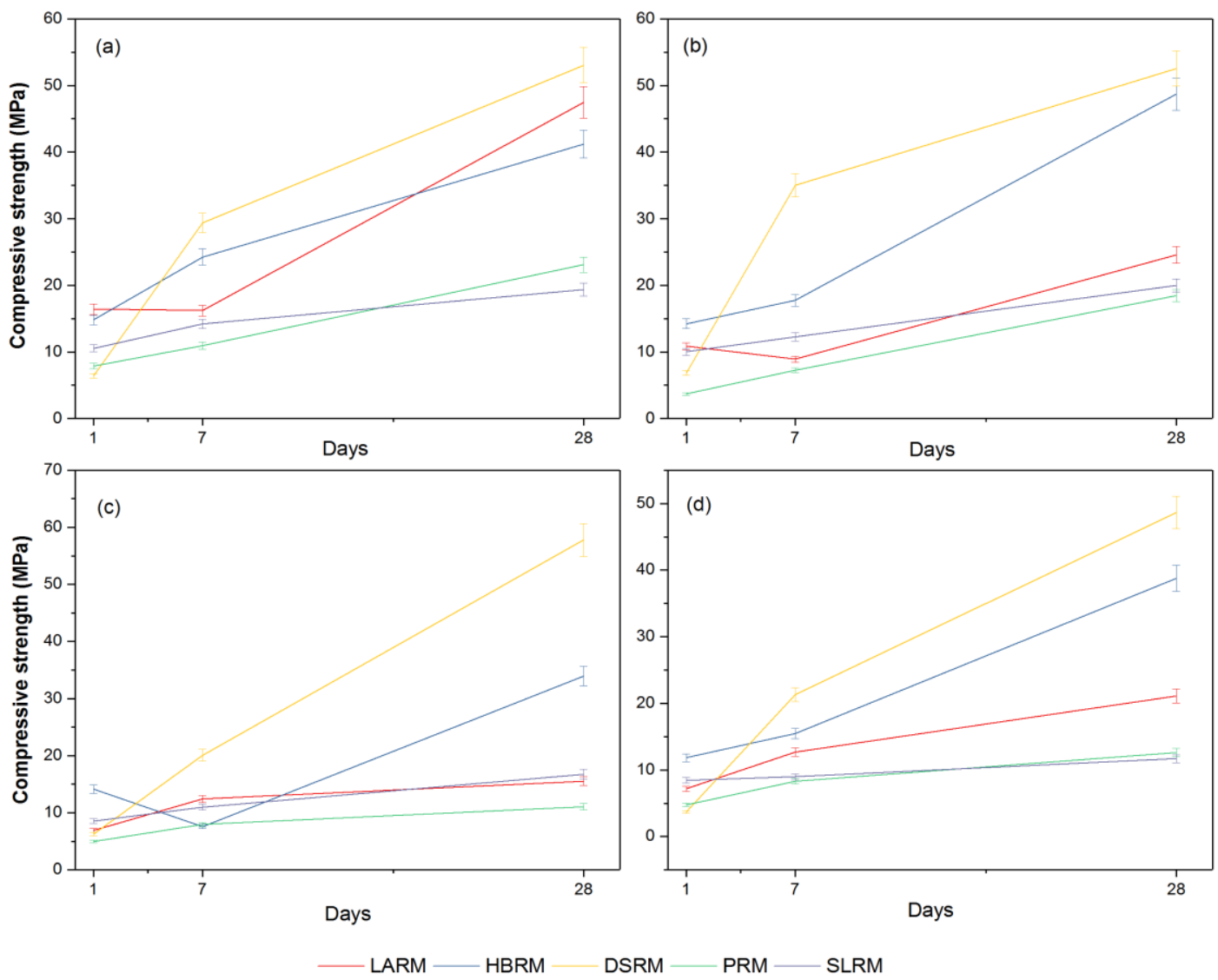

Fig. 4: Compressive strength variation at 1,7 and 28 days with increasing volume fraction (Vf) of microcapsules; (a) Control, (b) $8 \%$, (c) $16 \%$ and (d) $32 \%$ for LARM, HBRM, DSRM, PRM and SLRM.

\subsection{Base-line healing ability}

These initial compatibility results suggested three materials; HBRM, DSRM and SLRM and two microcapsule contents $8 \%$ and $16 \%$ by volume as most suitable for further exploration of the self-healing/selfrepairing ability of the composite materials. The intrinsic self-healing performance of the repair materials as measured by crack mouth healing and strength recovery helped further establish these repair materials as the ones with the greatest potential for the microcapsule enhancement studies. Indicative results for strength recovery are presented in Fig. 5. The results at 7 and 28 days showed HBRM $>$ DSRM $>$ SLRM $>$ PRM $>$ LA in order of the highest recovery potential. Macroscopic results of the crack mouth healing confirmed the mechanical recovery results as presented in Fig. 6.

\subsection{Improved healing ability and durability restoration}

The inclusion of microcapsules improved the crack closure and reduced the water absorption significantly compared to the cracked specimen. However larger volume fractions $(16 \%)$ were found to have slightly increased sorptivity coefficients with relatively large deviation in the results (Fig. 7). Yet a significant improvement in flexural strength was achieved for 2 of

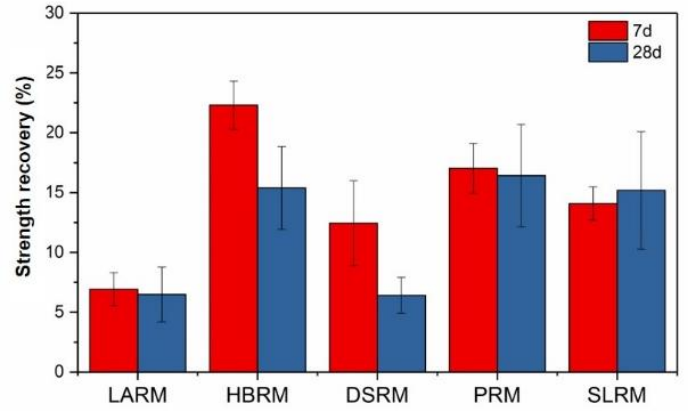

Fig. 5: Measured strength regain for different investigated materials according to initial age of cracking.

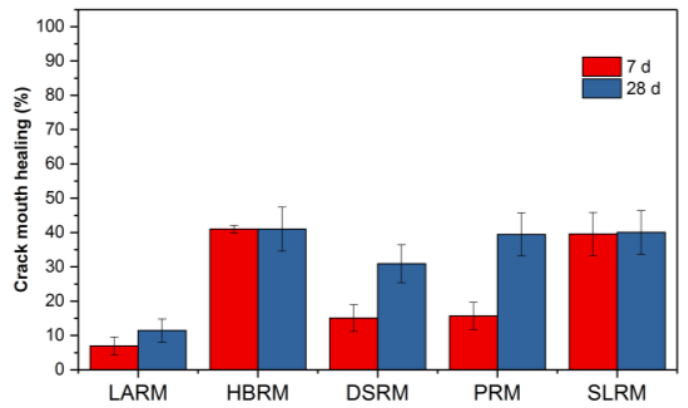

Fig. 6: Crack mouth healing for different investigated materials according to initial age of cracking.

the investigated materials (Fig. 8a), DSRM and SLRM even at the lowest investigated microcapsule content ( $8 \%$ Vf). This improvement outweighed the initial loss of 
flexural performance due to the addition of the microcapsules. It is implied that the intrinsic self-healing mechanism of the repair material itself is enhanced by the further interaction of the sodium silicate core released at the crack plane. The aforementioned results correlate with the macroscopic observation for crack mouth healing (Fig. 8b).

HBRM sees a total crack mouth healing value fluctuating around $45 \%$, marginally impaired by the higher microcapsule fraction as opposed to DSRM and SLRM. These show an improved crack closing potential linked to an increase in microcapsule concentration, approaching $75 \%$ and $65 \%$ for DSRM and SLRM, respectively, for $16 \%$ by volume microcapsule addition. The most pronounced effect can be observed on DSRM; where the intrinsic self-healing is improved almost four times as the microcapsule content doubles. It is interesting to see whether this macroscopic external crack mouth reduction links to a reduction in depth of the crack (Fig. 9). These measurements were performed at regular time intervals to monitor the progress of healing as indicated in Table 2. Results indicated that the addition of microcapsules increased the crack depth recovery; However, that improvement was only marginal. The greatest benefit seems to be in the reduction of crack opening and thus durability improvement rather than mechanical recovery.

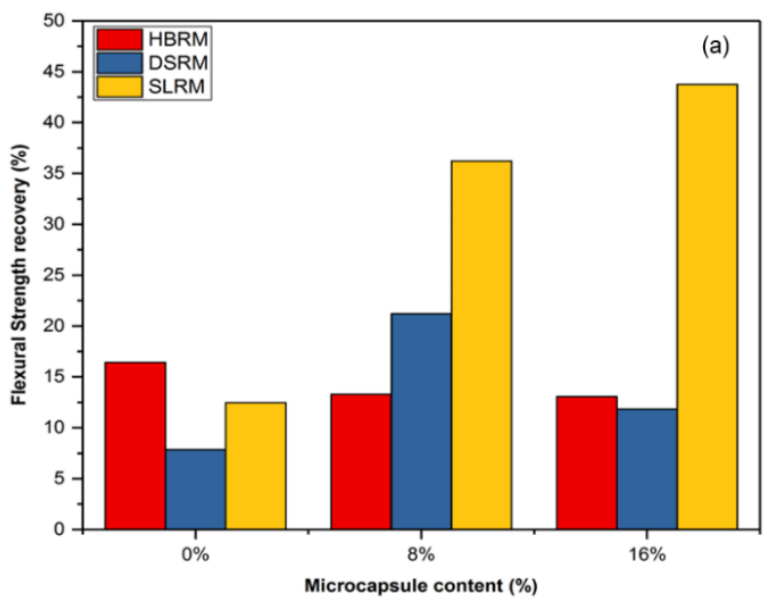

Fig 8 (a)

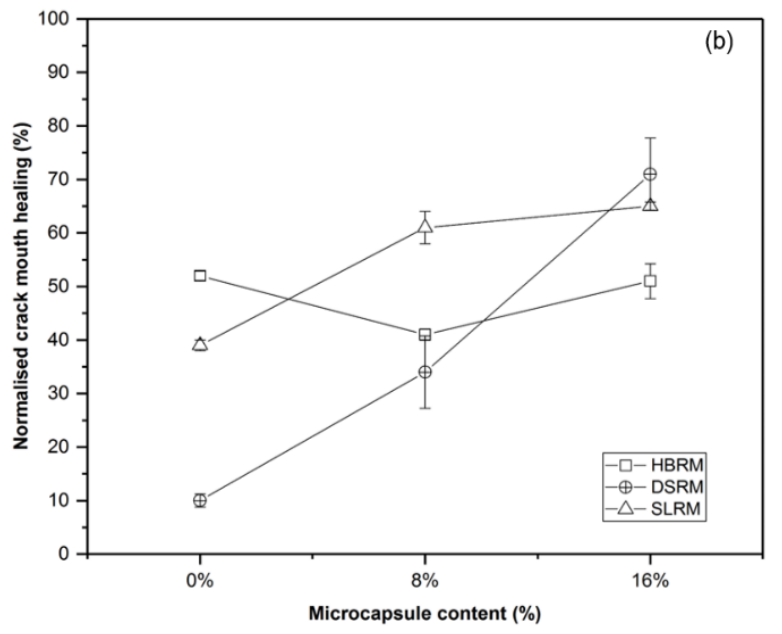

(b)

Fig. 8: (a) Measured strength regain for different investigated materials according microcapsule content; (b) Normalised crack healing achievable after 28 days healing period for samples with microcapsules cracked at 7 days.

\section{Conclusions}

The implementation of self-healing techniques as part of repair methodologies in extending the service life of concrete structures has great relevance as it can guarantee direct and timely ratification in the construction industry. The experimental results proved the compatibility of the proposed composite cement-based repair materials in terms of fresh properties and mechanical strength. Optimum self-healing additive fractions were selected for three end applications and further investigation on the in situ self-repair potential of these novel composite materials was carried through. The results showed improved crack-width and crack-depth reduction and recovery in permeability. The technical and practical considerations encountered during the project reported herein will assist the future development and accelerated commercialisation of targeted self-healing concrete applications using microencapsulated healing agents. 


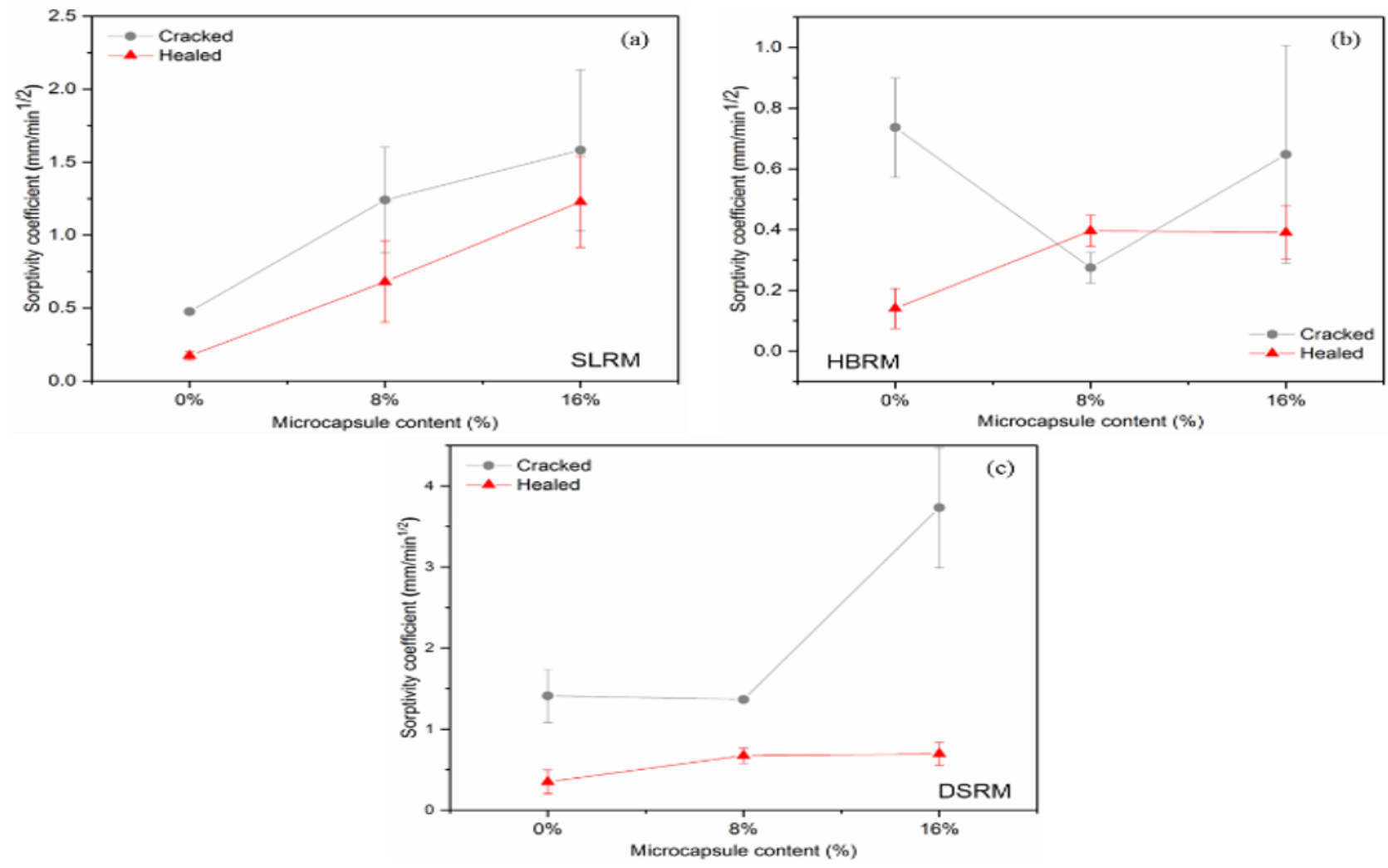

Fig. 7: Measured sorptivity coefficients for cracked, and healed samples; (a) SLRM, (b) HBRM and (c) DSRM.
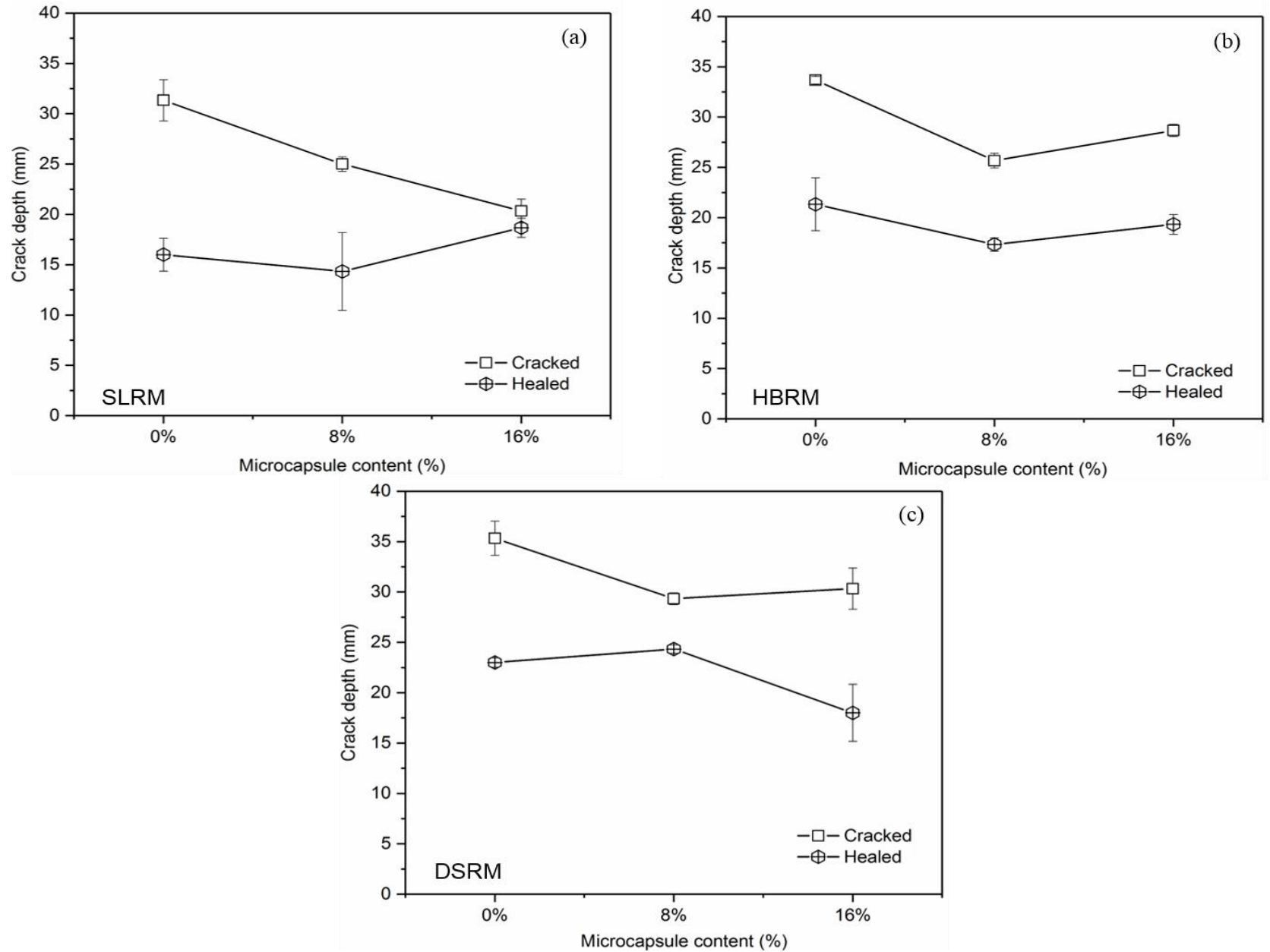

Fig. 9: : Crack depth variation with increasing volume fraction of microcapsules; (a) SLRM, (b) HBRM and (c) DSRM. 


\section{Acknowledgements}

Financial support from the UK Engineering and Physical Sciences Research Council (EPSRC-UK) for this study (Grant Ref. NMZJ/137, RG81609) is gratefully acknowledged.

\section{References}

1. A. Garbacz, L. Courard, and B. Bissonnette, Bull. Polish Acad. Sci. Tech. Sci. 61, (2013)

2. M. de Rooij, K. Van Tittelboom, N. De Belie, and E. Schlangen, Self-Healing Phenomena in Cement-Based Materials State-of-the-Art Report of RILEM Technical Committee 221-SHC: SelfHealing Phenomena in Cement-Based Materials (RILEM 2013, 2013)

3. D. Snoeck, K. Van Tittelboom, S. Steuperaert, P. Dubruel, and N. De Belie, J. Intell. Mater. Syst. Struct. 25, 13 (2012)

4. K. Van Tittelboom, N. De Belie, W. De Muynck, and W. Verstraete, Cem. Concr. Res. 40, 157 (2010)

5. K. Van Tittelboom and N. De Belie, Materials (Basel). 6, 2182 (2013)

6. T.-H. Ahn and T. Kishi, J. Adv. Concr. Technol. 8, 171 (2010)

7. K. Sisomphon, O. Copuroglu, and E. A. B. Koenders, Cem. Concr. Compos. 34, 566 (2012)

8. S. K. Ghosh, in Self-Healing Mater. Fundam. Des. Strateg. Appl., edited by S. K. Ghosh (WILEY-VCH Verlag GmbH \& Co. KGaA, 2009), pp. 1-25

9. J. P. Youngblood and N. R. Sottos, MRS Bull. 33, 732 (2008)

10. A. Kanellopoulos, P. Giannaros, D. Palmer, A. Kerr, and A. Al-Tabbaa, Smart Mater. Struct. 26, 045025 (2017)

11. P. Giannaros, A. Kanellopoulos, and A. AlTabbaa, Smart Mater. Struct. 25, 084005 (2016)

12. C. Litina and A. Al-Tabbaa, in 5th Int. Conf. SelfHealing Mater. (Durham, USA, 2015)

13. A. Kanellopoulos, P. Giannaros, and A. AlTabbaa, Constr. Build. Mater. 122, 577 (2016)

14. A. Al-Tabbaa, C. Litina, P. Giannaros, A. Kanellopoulos, and L. Souza, Constr. Build. Mater. 208, 669 (2019)

15. O. Teall, R. Davies, M. Pilegis, A. Kanellopoulos, T. Sharma, K. Paine, A. Jefferson, R. Lark, D. Gardner, and A. Al-Tabbaa, in Proc. 11th Fib Int. PhD Symp. Civ. Eng., edited by K. Maekawa, A. Kasuga, and J. Yamazaki (Tokyo, Japan, 2016), pp. 639-646

16. A. Kanellopoulos, T. S. Qureshi, and A. AlTabbaa, Constr. Build. Mater. 98, 780 (2015)

17. M. Pelletier, R. Brown, A. Shukla, and A. Bose,
Self-Healing Concrete with a Microencapsulated Healing Agent (Kingston, USA, 2011)

18. J. Gilford, M. M. Hassan, T. Rupnow, M. Barbato, A. Okeil, and S. Asadi, J. Mater. Civ. Eng. 886 (2013)

19. E. Mostavi, S. Asadi, M. M. Hassan, and M. Alansari, J. Mater. Civ. Eng. 27, 04015035 (2015)

20. R. Alghamri, A. Kanellopoulos, and A. AlTabbaa, Constr. Build. Mater. 124, 910 (2016) 